\title{
'Las Revoluciones Atlánticas: de Estados Unidos a Francia'. Un Ciclo de Mejora en el Aula para la asignatura de Historia General Contemporánea
}

\section{'Atlantic Revolutions: from the United States of America to France'. An Improvement Cycle in Classroom for the subject of Contemporary General History.}

BEgoÑA BARRERA LÓPEZ

ORCID: https://orcid.org/0000-0001-5574-8479

Universidad de Sevilla

Departamento de Historia Contemporánea

bbl@us.es

DOI: http://dx.doi.org/10.12795/9788447231003.003

Pp.: 67-85 


\section{Contexto}

Este Ciclo de Mejora en el Aula (en adelante CIMA), titulado "Las Revoluciones Atlánticas: de Estados Unidos a Francia", fue diseñado en el marco del Curso General de Docencia Universitaria de la Universidad de Sevilla. En su planificación e implementación han influido muchos factores, entre ellos, y de forma muy destacada, la situación de semipresencialidad a la que nos hemos visto abocados en el curso 2020/21. No obstante, he optado por no centrar el relato del CIMA2 en las dificultades que esta circunstancia ha supuesto, pues creo que estas serán conocidas por la mayoría de mis compañeros docentes. Por ello, solo haré alusión a estos impedimentos si considero que han influido de forma destacada en el desarrollo de las sesiones. Otro factor esencial en la preparación de este CIMA2 ha sido la experiencia del CIMA1, que se aplicó durante la primera semana de confinamiento del mes de marzo de 2020 en una asignatura muy similar a la que ha servido para poner en práctica el CIMA2.

Este CIMA2 fue diseñado para ser implementado en la asignatura Historia General Contemporánea, que se imparte en el Primer curso del Grado de Geografía, en la Facultad de Geografía e Historia de la Universidad de Sevilla. Se trata de una asignatura "Fundamental", que normalmente cursan en torno a cuarenta alumnos cada año. El conocimiento que los alumnos suelen tener de esta asignatura es escaso, a pesar de haberla cursado en 10 de Bachillerato una gran mayoría. Las clases impartidas han tenido lugar en un aula de la Facultad de Geografía e Historia, un lugar poco propicio para cualquier tipo de innovación docente por la disposición de los asientos corridos y por el estrecho espacio que queda entre las bancas y la pared para desplazarse alrededor del aula. No obstante, dada la situación de semipresencialidad antes aludida y el protocolo COVID19, durante la implementación del CIMA esta disposición no ha supuesto un gran inconveniente, como sí había ocurrido en años anteriores. El 
equipamiento técnico, por otro lado, facilitó enormemente el desarrollo de las sesiones.

El CIMA2 fue implementado en la segunda mitad del mes octubre de 2020. Para entonces, la asignatura ya llevaba una semana de impartición, de modo que alumnos y profesora ya nos conocíamos y habíamos establecido un cierto clima de familiaridad.

\section{Diseño previo del CIMA}

\section{Mapa de contenidos}

Uno de los problemas que diagnostiqué al autoevaluar mi modelo metodológico habitual era mi incapacidad para abordar en clase todos los contenidos que abarcaba el programa de la asignatura. En este CIMA2 puse en práctica una nueva forma de pensar y expresar aquello que quería que los estudiantes aprendiesen: el mapa de contenidos. La elaboración de este mapa exigió, en primer lugar, una reflexión sobre la relevancia de los contenidos que normalmente había abordado en clase ¿Eran todos igual de importantes? ¿Había datos o referencias anecdóticas que solo incorporaba a mi discurso por pura rutina docente? ¿Estaba ayudando a mis alumnos a reflexionar sobre los problemas principales de la materia o estos quedaban eclipsados en una nube de conceptos accesorios?

Esta reflexión me ayudó a escoger aquellos contenidos que me parecían más significativos y a plantear temas que normalmente se imparten por separado (la Independencia de los Estados Unidos de América y la Revolución Francesa) como un mismo problema historiográfico, siguiendo así las nuevas tendencias dentro de la propia disciplina (Gould y Onuf, 2015; Klooster, 2018). Con ellos elaboré un mapa de contenidos (figura 1) en el que se muestra tanto la diferente naturaleza de estos contenidos (conceptual, procedimental, metadisciplinar y actitudinal), como la relación que se establece entre ellos. Al estar referido a un

Ciclos de Mejora en el Aula (2020). Experiencias de Innovación Docente de la US Esta obra se distribuye con la licencia Creative Commons 
periodo histórico, el mapa, además de mostrar una interrelación entre contenidos, expone el sentido procesual o secuencial propio del fenómeno histórico al que se refiere.

Este mapa conceptual se estructura a partir de una pregunta fundamental, referida a un problema social relacionado con la materia (García Díaz, Porlán y Navarro, 2017), y tres preguntas subsecuentes derivadas de la primera. El sentido interpelativo de la pregunta debe funcionar como un resorte que active la curiosidad de los alumnos y comience a movilizar sus ideas previas (Finkel, 2008). A su vez, estas cuatro preguntas sirven para organizar los contenidos, que a su vez aparecen jerarquizados en función de su importancia. La estructura cuatripartita fue la base de la planificación de una secuencia de actividades en cuatro sesiones distintas.

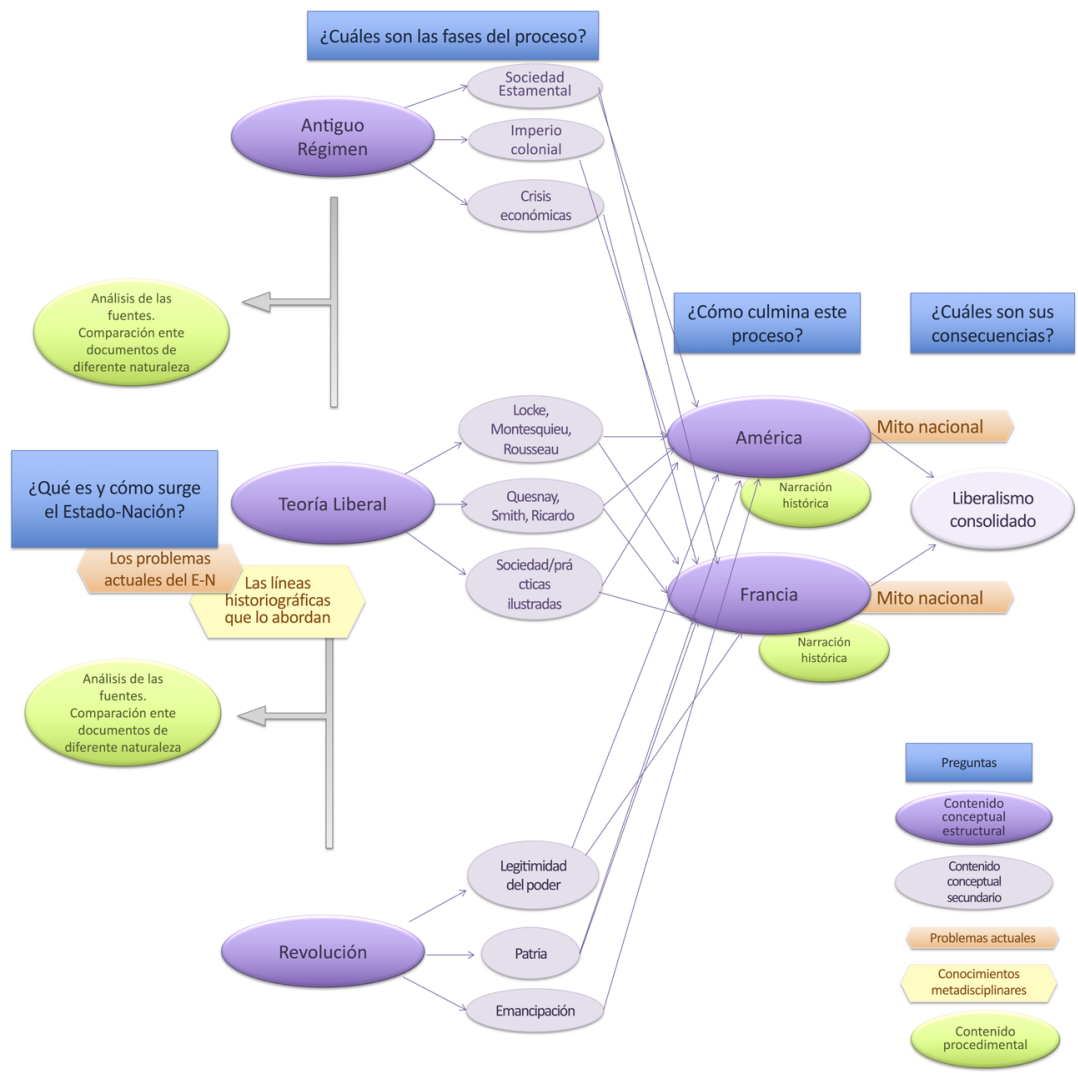

Figura 1. Mapa de contenidos del CIMA 2

Ciclos de Mejora en el Aula (2020). Experiencias de Innovación Docente de la US Esta obra se distribuye con la licencia Creative Commons 


\section{Modelo metodológico}

Mi modelo habitual había sido, hasta el inicio de la formación en el CGDU, la clase magistral tradicional. Si la sesión duraba 120 minutos, dejaba unos 5 o 10 primeros minutos de cortesía mientras preparaba el Power Point. Tras ello, me limitaba a explicar el tema durante el resto de la clase, intercalando mi exposición con breves videos o con actividades cortas de comentario de textos. Frente a ello, el diseño de este CIMA2 se basa en un nuevo modelo metodológico en el que la predominancia de la clase magistral ha dejado paso a un mayor protagonismo de los estudiantes en la construcción de su propio conocimiento (De Alba y Porlán, 2017). Este nuevo modelo metodológico comienza con el planteamiento de un problema, prosigue con una secuencia de actividades de contraste (que ocupan el grueso del CIMA) y finaliza con unas conclusiones que recogen tanto las ideas de los alumnos como las orientaciones/correcciones de la docente. Su propósito es fomentar el "aprendizaje crítico-natural" (Bain, 2007), en el que los estudiantes se sienten implicados en la construcción de su propio conocimiento, al tiempo que se van introduciendo en los problemas de la disciplina que están estudiando.

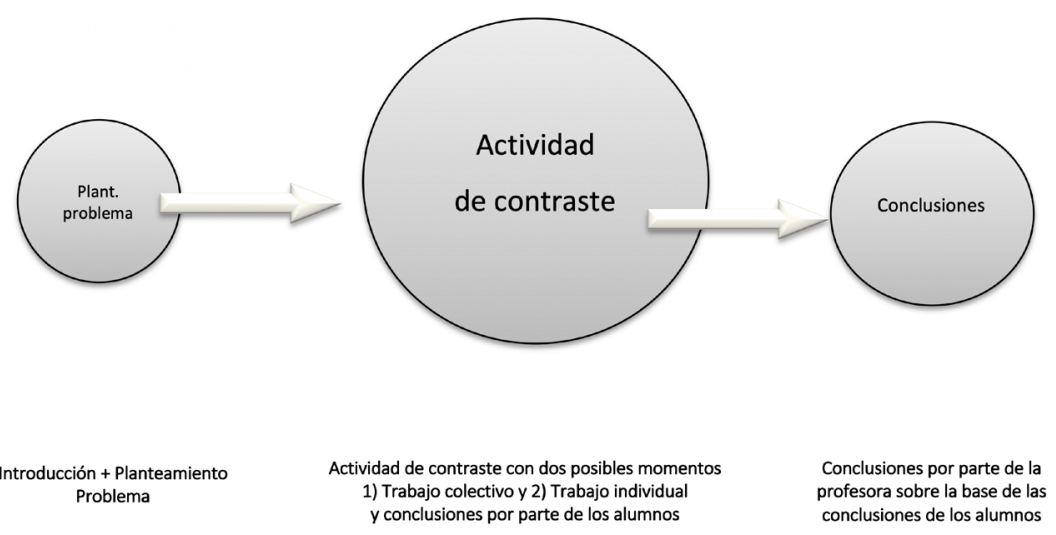

Figura 2. Modelo metodológico

Ciclos de Mejora en el Aula (2020). Experiencias de Innovación Docente de la US Esta obra se distribuye con la licencia Creative Commons 


\section{Cuestionario de ideas iniciales}

El cuestionario ha sido una herramienta fundamental para conocer los modelos mentales previos de los alumnos (Rivero y Porlán, 2017) y para valorar su conocimiento tras la aplicación del CIMA. Las cinco cuestiones que compusieron este cuestionario fueron las mismas que organizaban el mapa de contenidos. Así, los alumnos pudieron percibir la vinculación que existía entre todas las tareas de la actividad y esta coherencia les dio seguridad.

El cuestionario se realizó mediante la aplicación Google Forms, lo que permitió mayor flexibilidad a la hora de responder y redujo la presión que la vigilancia del profesor en el aula suele suponer para los alumnos. Además, dado que este CIMA se desarrolló en una situación de docencia semi-presencial, el uso de Google Forms permitió que tanto los estudiantes que esa semana tenían clase presencial, como los que la tenía virtual, pudieran participar de la actividad. Estas fueron las preguntas abiertas que conformaron el cuestionario:

1. ¿Qué son y cuándo surgen los Estados-nación? ¿Qué características crees que compartimos quienes vivimos en este Estado-nación?

2. ¿En qué se diferencia nuestra relación actual con el poder de la que tenía un individuo en el siglo XVIII?

3. ¿Por qué crees que los habitantes de las colonias americanas de Gran Bretaña querían independizarse de la metrópoli?

4. ¿Por qué crees que a lo ocurrido en Francia en 1789 se le llama "Revolución"?

5. ¿Qué consecuencias crees que tiene para el mundo actual la independencia de Estados Unidos y la Revolución Francesa?

Ciclos de Mejora en el Aula (2020). Experiencias de Innovación Docente de la US Esta obra se distribuye con la licencia Creative Commons 
A continuación se detalla la secuencia de actividades diseñada para este CIMA2, que comprende cuatro sesiones, de 110 minutos cada una.

\begin{tabular}{|l|l|l|l|}
\hline $\begin{array}{l}\text { Fase previa } \\
\text { Ideas de los alumnos }\end{array}$ & No 0 & $\begin{array}{l}\text { Nombre: Cuestionario en } \\
\text { Google Forms }\end{array}$ & $\begin{array}{l}\text { Tiempo: 3 días } \\
\text { previos al inicio del } \\
\text { CIMA }\end{array}$ \\
\hline La fase previa consiste en la realización del cuestionario por parte de los alumnos. \\
\hline \multicolumn{3}{|c|}{ PRIMERA SESIÓN } \\
\hline $\begin{array}{l}\text { 1a fase (planteamiento } \\
\text { problema) }\end{array}$ & № 1 & $\begin{array}{l}\text { Nombre: ¿Qué son y } \\
\text { cuándo/cómo surgen los } \\
\text { Estado-Nación? }\end{array}$ & $\begin{array}{l}\text { Tiempo: } 10 \text { min } \\
\text { aprox. }\end{array}$ \\
\hline
\end{tabular}

La primera parte de la sesión está dedicada a la presentación del problema principal, ¿Qué son y cuándo/cómo surgen los Estados-Nación? La pregunta se proyecta en la pantalla y se comparte por Collaborate mientras se explica la secuencia de actividades que se va a desarrollar en las siguientes cuatro sesiones, así como las herramientas que vamos a utilizar (diario personal de las sesiones, dosieres de fuentes, etc.). También se expone el mapa de contenidos y se explica brevemente la relación que existe entre cada parte de la actividad y la estructura de este mapa. 10 min.

\begin{tabular}{|l|l|l|l|}
\hline $\begin{array}{l}\text { 2a fase (actividad de } \\
\text { contraste) }\end{array}$ & № 2 & $\begin{array}{l}\text { Nombre: ¿Por qué la } \\
\text { definición del Estado y de la } \\
\text { nación supone un conflicto? }\end{array}$ & $\begin{array}{l}\text { Tiempo: } 100 \text { min } \\
\text { aprox. }\end{array}$ \\
\hline
\end{tabular}

La segunda parte de la sesión está dedicada a responder a la subpregunta ¿Por qué la definición del Estado y de la nación supone un conflicto?, que se proyecta así formulada en la pantalla de clase y de Collaborate. Esta pregunta está referida a los casos históricos y actuales que se recogen en un dosier que el docente prepara previamente a partir de fuentes hemerográficas relativas a los problemas del Estado-Nación [Dosier 1]. La actividad, por tanto, consiste en leerlas y tomar notas individuales que den respuesta a la pregunta. Sus respuestas y otras ideas que surjan son anotadas en el diario de clase de cada alumno, en el que ya deben figurar las respuestas al cuestionario inicial. Se trata, por tanto, de una actividad individual, que pueden hacer tanto los que estén en clase como los que estén conectados por Collaborate. Las dudas que surjan son atendidas de forma presencial y por medio del Collaborate durante la sesión. 80 min. aprox.

La última parte de la sesión está dedicada a una puesta en común de las ideas individuales, lo cual permite completar las anotaciones de cada alumno. 20 min. aprox.

\section{SEGUNDA SESIÓN}

Ciclos de Mejora en el Aula (2020). Experiencias de Innovación Docente de la US Esta obra se distribuye con la licencia Creative Commons Reconocimiento-NoComercial-SinObraDerivada Internacional (CC BY-NC-ND 4.0.) 


\begin{tabular}{|l|l|l|l}
\hline $\begin{array}{l}\text { 2a fase (actividad de } \\
\text { contraste) }\end{array}$ & № 2 & $\begin{array}{l}\text { Nombre: ¿Cómo se } \\
\text { transforma la relación de los } \\
\text { individuos con el poder a } \\
\text { finales del siglo XVIII? }\end{array}$ & Tiempo: 110 min. \\
\hline
\end{tabular}

La primera parte de la clase consiste en la visualización de dos videos: uno corresponde a la serie John Adams, y describe la vida en las colonias británicas hacia 1770; el otro pertenece a la serie Histoire d'une Revolution, y narra la vida en París en los años previos a 1789. Entre los dos, $30 \mathrm{~min}$. aproximadamente. A continuación, trabajan con un nuevo dosier de fuentes en los que se recogen algunos textos de personajes que aparecen en los videos visualizados ('Common Sense' de Thomas Payne, los 'Cahiers de Doleances', 'El Tercer Estado' del Abate Sièyes') y que se refieren a la vida política y social durante el Antiguo Régimen. Junto con ellos, se incluyen tres textos breves de teoría política clásica (John Locke, Montesquieu y Rousseau) [Dosier 2]. La actividad que tienen que hacer individualmente consiste en responder, citando las fuentes del dosier, a la pregunta ¿Cómo se transforma la relación de los individuos con el poder a finales del siglo XVIII? Para ello, deben comparar los argumentos del primer grupo de textos con los del segundo.

Las conclusiones que extraigan deben ser incluidas en su diario. Mientras las realizan, el docente les orienta desde Collaborate o en el aula. Esta segunda actividad ocupa el resto de la sesión, 80 min. aprox.

TERCERA SESIÓN

\begin{tabular}{l|l|l|l}
\hline $\begin{array}{l}\text { 2a fase (actividad de } \\
\text { contraste) }\end{array}$ & № 3 & $\begin{array}{l}\text { Nombre: América y Francia } \\
\text { en revolución. }\end{array}$ & $\begin{array}{l}\text { Tiempo: } 110 \text { min. } \\
\text { aprox. }\end{array}$ \\
\hline
\end{tabular}

La metodología de esta sesión es la misma:

De nuevo, la primera parte de la clase consiste en la visualización de dos videos: el primero, de la serie John Adams, corresponde a los debates sobre la independencia que tuvieron lu-gar en los Congresos Nacionales; el segundo, también de la serie Histoire d'une Revolution, se refiere a la toma de la Bastilla. Entre los dos, 30 min. aproximadamente. 
A continuación, los alumnos trabajan con un nuevo dosier de fuentes en el que se recogen algunos textos que hacen referencia a episodios vistos en los videos (Declaración de Inde-pendencia 1776, Constitución de los Estados Unidos de 1787, Juramento del Juego de la Pelota de 1789, Primera Constitución Francesa de 1789) [Dosier 3]. Esta vez, el dosier está divido claramente en fuentes relativas al caso americano y al caso francés. En vez de a una pregunta, deben responder a dos, citando las fuentes que se les ha dado: ¿Por qué querían independizarse los habitantes de las colonias americanas? ¿Por qué a lo ocurrido en Fran-cia se le llama revolución? Igual que en la sesión anterior, las conclusiones que extraigan las deben incluir en su diario. No deben tener más de dos carillas de extensión. Mientras las realizan, el docente les orienta desde Collaborate o en el aula. Esta segunda actividad ocupa el resto de la sesión, $80 \mathrm{~min}$. aprox.

Antes de acabar la sesión, se propone que, quien quiera, puede exponer las conclusiones de los dos ejercicios en una breve presentación durante la sesión siguiente. Esta presentación debe incluir tanto las respuestas de las actividades 1,2 y 3, como una breve reflexión sobre las consecuencias que cree que estos acontecimientos tuvieron en la historia.

\begin{tabular}{|l|l|l|l} 
3a fase (conclusiones) & № 1 & $\begin{array}{l}\text { Nombre: ¿Qué consecuencias } \\
\text { tuvieron las revoluciones } \\
\text { americana y francesa? }\end{array}$ & Tiempo: $110 \mathrm{~min} .$. \\
\hline
\end{tabular}

La clase comienza con las exposiciones de las conclusiones de aquellos estudiantes que ha-yan decidido preparar una presentación. Tras la ronda de presentaciones, hay un turno de preguntas y comentarios para que el resto de los estudiantes puedan mostrar su parecer so-bre el trabajo de sus compañeros. Esta actividad ocupará como mucho 60 o 75 min.

Tras ello, se visualizan dos videos, esta vez más breves ( 5 min. aproximadamente cada uno) que muestran las celebraciones del Día de la Independencia-4 julio en Estados Unidos y la fiesta nacional francesa del 14 de julio. Estos videos sirven para enmarcar las conclusiones finales, que corren a cargo del docente. Se trata de partir del mito nacional para señalar la importancia que tuvieron los procesos revolucionarios tanto en el momento que se produje-ron como en el desarrollo posterior de los Estados nacionales de EEUU y Francia. Estas conclusiones se elaborarán con las notas tomadas durante las tres sesiones anteriores. Con todo ello se elabora un documento y una breve presentación que los alumnos tienen dispo-nible en Enseñanza Virtual en los días posteriores al CIMA. 


\section{Descripción y análisis de las clases del segundo CIMA}

\section{Relato de las sesiones}

El CIMA "Las Revoluciones Atlánticas: de Estados Unidos a Francia" se implementó las semanas del 19 al 30 de octubre de 2020. La actividad inicial (0), que consistía en la respuesta al cuestionario, contó con muy poca participación. Hasta que la finalidad del cuestionario no fue explicada con detenimiento y de forma presencial/streaming en clase, subrayando la necesidad de su implicación en esta tarea inicial para el correcto desarrollo de la secuencia de actividades, los alumnos no le dieron la importancia que merecía. Una vez lo comprendieron, un mayor número de estudiantes accedió a realizar el cuestionario (18 de 41 matriculados).

La primera sesión se desarrolló con menos problemas de los previstos, aunque sí hubo dificultades. La primera que se puede señalar estuvo relacionada con la realización del trabajo de forma individual. Los estudiantes parecían tener muy asociado el trabajo en el aula al trabajo en grupo, y por tanto buscaban interpelarse y comparar sus impresiones. Como el protocolo sanitario para el curso 2020/21 marca la necesidad de guardar la distancia de seguridad, al comenzar la primera actividad de contraste les indiqué que podían comentar sus opiniones con sus compañeros sin problema, siempre que lo hicieran mediante el Chat de Collaborative. La segunda dificultad tuvo que ver con las explicaciones que yo fui aportando a lo largo de la clase. Dado que los alumnos manifestaban frecuentemente dudas comunes, decidí intervenir en varias ocasiones para hacer aclaraciones. Desde el principio fui consciente de que estaba aportando más información de la necesaria y que me superaba la ansiedad por ver resuelto el ejercicio en el tiempo previsto. Tras la clase reflexioné sobre la 
conveniencia de seguir manteniendo estas explicaciones (pues resultaron fundamentales) pero de hacerlo limitándome a mí misma el tiempo y los conceptos ("solo responder a lo que hayan preguntado, y no hablar más de un minuto", me propuse).

La segunda sesión ya suponía el trabajo con fuentes historiográficas de más dificil interpretación. Por tanto, como era previsible, los alumnos necesitaron más asistencia para comprender y analizar los textos. Según se fue desarrollando la sesión, pedí a algunos de ellos que formulasen sus preguntas en voz alta, pues me pareció que sus dudas podían ser compartidas. Traté de mantener la regla autoimpuesta de hablar poco y solo para lo fundamental, aunque fue complicado y seguramente se percibió como algo forzado. Por otra parte, la pregunta a la que tenían que dar respuesta con los materiales del dosier 2 era verdaderamente compleja, tal vez la más dificil de todo el CIMA. Así se lo hice saber y, paradójicamente, esto pareció animarlos a trabajar con más intensidad lo que quedaba de sesión. Alguno de ellos ya mostró desde temprano su deseo de exponer y "debatir" lo que habían anotado en sus cuadernos. A estos alumnos, les propuse que aprovecharan la tarde para revisar sus anotaciones, si es que ya habían decidido que realizarían la presentación final voluntaria.

La tercera sesión comenzó con un problema técnico que retrasó el inicio de la actividad 30 minutos. Sin embargo, al parecer los alumnos encontraron más sencillo el análisis de las fuentes historiográficas del dosier 3. En esta sesión se percibió que ya se habían familiarizado con la dinámica y, de hecho, trabajaron más individualmente que en las sesiones anteriores: hubo menos actividad en Chat y menos dudas a la hora de desarrollar sus conclusiones provisionales en su diario. En todo caso, es importante señalar que el subgrupo que estuvo presente en la sesiones tercera y cuarta era diferente del subgrupo de las primeras dos sesiones. Ambos subgrupos habían mostrado, desde el comienzo de las clases, un comportamiento y una dinámica interna bastante diferentes.

Ciclos de Mejora en el Aula (2020). Experiencias de Innovación Docente de la US Esta obra se distribuye con la licencia Creative Commons 
En la cuarta sesión, dedicada a la exposición de las conclusiones individuales, hubo una alta participación e interés. En las presentaciones individuales se apreciaron algunas dificultades metodológicas y conceptuales que yo me fui apuntando para comentarlas en la actividad final. Estas dificultades tenían que ver con la capacidad de síntesis y de exposición (metodológicas) y con el empleo de conceptos específicos de la historia política. Los once alumnos que realizaron sus exposiciones presenciales y por streaming compartían, en términos generales, los mismos errores y un interés semejante por algunos de temas tratados. La actividad final, referida a la construcción de los mitos nacionales estadounidense y francés sobre la base de los procesos históricos que habíamos trabajado, pareció entusiasmarles. Las conclusiones por mi parte fueron más breves de lo previsto, pues les percibí cansados y tal vez ya un poco saturados del tema. Tal y como ya había hecho en el CIMA 1, les remití a un breve documento que les subiría a Enseñanza Virtual en el que encontrarían estas conclusiones (suyas y mías) más desarrolladas. Finalmente, les recordé que a partir de entonces y hasta el domingo tendrían disponible el cuestionario para volver a cumplimentarlo.

\section{Evaluación del aprendizaje}

El cuestionario con las cinco preguntas arriba recogidas fue respondido por los estudiantes antes de comenzar y al final del CIMA. Sus resultados han permitido realizar las escaleras de aprendizaje relativas a cada pregunta, así como una tabla de progreso individual. Su resultado arroja una imagen muy significativa de cuáles son los principales obstáculos de aprendizaje del grupo, que se encuentran en su mayoría en el primer escalón y corresponden, por tanto, a la comprensión de los conceptos más básicos.

Ciclos de Mejora en el Aula (2020). Experiencias de Innovación Docente de la US Esta obra se distribuye con la licencia Creative Commons 
ESCALERA DE APRENDIZAJE CORRESPONDIENTE A LA PREGUNTA 1. ¿QUÉ SON Y CUÁNDO SURGEN LOS ESTADOS-NACIÓN? ¿QUÉ CARACTERÍSTICAS CREES QUE COMPARTIMOS QUIENES VIVIMOS EN ESTE ESTADO-NACIÓN?

\section{C) Lo interpreta como un proceso histórico complejo e identifica correctamente los rasgos esenciales. \\ $0 \% \rightarrow 38.89 \%$}

B) Alude a su origen histórico y naturaleza procesual, aunque no lo localiza correctamente y solo cita algunos atributos esenciales.

$27.78 \% \rightarrow 50 \%$

A) No razona el origen histórico ni su evolución; no cita atributos esenciales.

$77.78 \% \rightarrow 11.11 \%$

Obstáculo: razonamiento histórico y naturaleza procesual.
Obstáculo: principales características asociadas al fenómeno

ESCALERA DE APRENDIZAJE CORRESPONDIENTE A LA PREGUNTA 2. ¿EN QUÉ SE DIFERENCIA NUESTRA RELACIÓN ACTUAL CON EL PODER DE LA QUE TENÍA UN INDIVIDUO EN EL SIGLO XVIII?

c) Identifica correctamente los rasgos esenciales que diferencian al Antiguo y Nuevo Régimen.

$$
0 \% \rightarrow 33.33 \%
$$

Ciclos de Mejora en el Aula (2020). Experiencias de Innovación Docente de la US Esta obra se distribuye con la licencia Creative Commons 
B) Señala algunos rasgos que diferencian al Antiguo y Nuevo Régimen, pero de manera desordenada y/o insuficiente. $33.33 \% \rightarrow 55.56 \%$

\begin{abstract}
A) Alude de forma desordenada a rasgos del Antiguo y Nuevo Régimen sin identificarlos correctamente.
\end{abstract}

$72.22 \% \rightarrow 16.67 \%$

ESCALERA DE APRENDIZAJE CORRESPONDIENTE A LA PREGUNTA 3. ¿POR QUÉ CREES QUE LOS HABITANTES DE LAS COLONIAS AMERICANAS DE GRAN BRETAÑA QUERIAN INDEPENDIZARSE DE LA METRÓPOLI?

Obstáculo: identificación de los elementos específicos asociados a las relaciones de poder.
Obstáculo:

conceptualización básica

del Antiguo y Nuevo

Régimen.
B) Señala la naturaleza multicausal pero no alude a los factores principales. O la inversa.

$22.22 \% \rightarrow 50 \%$

A) Simplifica el acontecimiento histórico y/o se refiere solo a las más superficiales de sus causas.

$77.78 \% \rightarrow 11.11 \%$
C) Identifica la naturaleza multicausal y compleja del fenómeno señalando los factores más importantes de forma ordenada.

$0 \% \rightarrow 44.44 \%$

Obstáculo: identificación y jerarquización de los factores implicados en el proceso.

Ciclos de Mejora en el Aula (2020). Experiencias de Innovación Docente de la US Esta obra se distribuye con la licencia Creative Commons 
ESCALERA DE APRENDIZAJE CORRESPONDIENTE A LA PREGUNTA 4. ¿POR QUÉ CREES QUE A LO OCURRIDO EN FRANCIA EN 1789 SE LE LLAMA "REVOLUCIÓN"?

\author{
C) Define correctamente el \\ término y lo relaciona con los \\ acontecimientos históricos, \\ añadiendo ejemplos \\ apropiados.
}

$0 \% \rightarrow 33.33 \%$

B) Define correctamente el término, pero no lo relaciona con los acontecimientos históricos. O a la inversa. $33.33 \% \rightarrow 55.56 \%$

\section{A) Ni define} correctamente el término ni localiza adecuadamente los hechos históricos a los que se refiere.

$66.67 \% \rightarrow 16.67 \%$
Obstáculo: identificación

la idea de ruptura

y localización en su

horizonte temporal

aproximado.

ESCALERA DE APRENDIZAJE CORRESPONDIENTE A LA PREGUNTA 5. ¿QUÉ CONSECUENCIAS CREES QUE TIENE PARA EL MUNDO ACTUAL LA INDEPENDENCIA DE ESTADOS UNIDOS Y LA REVOLUCIÓN FRANCESA?

C) Alude correctamente a los conceptos básicos de la extensión del liberalismo y lo relaciona con las democracias actuales

$0 \% \rightarrow 44.44 \%$

Ciclos de Mejora en el Aula (2020). Experiencias de Innovación Docente de la US Esta obra se distribuye con la licencia Creative Commons 
B) Alude a sus implicaciones inmediatas, pero no lo relaciona correctamente con la actualidad. $\mathrm{O}$ a la inversa. $33.33 \% \rightarrow 44.44 \%$

Obstáculo: conexión de las consecuencias de los procesos revolucionarios con la realidad sociopolítica actual.
A) Ni alude correctamente a sus implicaciones históricas ni lo relaciona adecuadamente con la actualidad.

$66.67 \% \rightarrow 16.67 \%$
Obstáculo: identificación

de las consecuencias

más importantes del

acontecimiento.

\begin{tabular}{|c|c|c|c|c|c|c|c|c|c|c|c|c|c|c|c|}
\hline & \multicolumn{3}{|c|}{ Pregunta 1} & \multicolumn{3}{|c|}{ Pregunta 2} & \multicolumn{3}{|c|}{ Pregunta 3} & \multicolumn{3}{|c|}{ Pregunta 4} & \multicolumn{3}{|c|}{ Pregunta 5} \\
\hline & $A$ & B & C & A & B & C & A & B & C & A & B & C & $A$ & B & $\mathrm{C}$ \\
\hline Estudiante 1 & & $\uparrow 1$ & & & $\uparrow 1$ & & & & $\uparrow 2$ & & & $\uparrow 2$ & $=$ & & \\
\hline Estudiante 2 & & $\uparrow 1$ & & $=$ & & & & $\uparrow 1$ & & & $=$ & & $=$ & & \\
\hline Estudiante 3 & & $=$ & & & $=$ & & & $=$ & & & & $\uparrow 1$ & & $\uparrow 1$ & \\
\hline Estudiante 4 & & $\uparrow 1$ & & & $=$ & & $=$ & & & $=$ & & & $=$ & & \\
\hline Estudiante 5 & & $\uparrow 1$ & & & $\uparrow 1$ & & & & $\uparrow 2$ & & & $\uparrow 2$ & & & $\uparrow 2$ \\
\hline Estudiante 6 & & & $\uparrow 2$ & & $\uparrow 1$ & & & $\uparrow 1$ & & & $=$ & & & $=$ & \\
\hline Estudiante 7 & & & $\uparrow 1$ & & & $\uparrow 1$ & & & $\uparrow 1$ & & $=$ & & & & $\uparrow 1$ \\
\hline Estudiante 8 & & $\uparrow 1$ & & & $\uparrow 1$ & & & $\uparrow 1$ & & & & $\uparrow 2$ & & $\uparrow 1$ & \\
\hline Estudiante 9 & & & $\uparrow 2$ & & $\uparrow 1$ & & & $\uparrow 1$ & & & & $\uparrow 2$ & & $\uparrow 1$ & \\
\hline Estudiante 10 & & & $\uparrow 2$ & & & $\uparrow 2$ & & & $\uparrow 2$ & & $\uparrow 1$ & & & & $\uparrow 2$ \\
\hline Estudiante 11 & & $\uparrow 1$ & & $=$ & & & & & $\uparrow 2$ & & $\uparrow 1$ & & & & $\uparrow 2$ \\
\hline Estudiante 12 & & $\uparrow 1$ & & & $\uparrow 1$ & & & $\uparrow 1$ & & & & $\uparrow 2$ & & $=$ & \\
\hline Estudiante 13 & & & $\uparrow 1$ & & & $\uparrow 1$ & & & $\uparrow 1$ & & $=$ & & & & $\uparrow 1$ \\
\hline Estudiante 14 & & & $\uparrow 1$ & & $\uparrow 1$ & & & & $\uparrow 1$ & & $=$ & & & & $\uparrow 1$ \\
\hline Estudiante 15 & & $\uparrow 1$ & & & & $\uparrow 1$ & & $\uparrow 1$ & & & & $\uparrow 2$ & & $\uparrow 1$ & \\
\hline Estudiante 16 & & & $\uparrow 2$ & & $\uparrow 1$ & & & $\uparrow 1$ & & & $\uparrow 1$ & & & & $\uparrow 1$ \\
\hline Estudiante 17 & $=$ & & & & $\uparrow 1$ & & & $\uparrow 1$ & & & 个1 & & & $=$ & \\
\hline Estudiante 18 & $=$ & & & $=$ & & & $=$ & & & $=$ & & & & $\uparrow 1$ & \\
\hline
\end{tabular}

Cuadro de evolución de los alumnos

Ciclos de Mejora en el Aula (2020). Experiencias de Innovación Docente de la US Esta obra se distribuye con la licencia Creative Commons 


\section{Evaluación del Ciclo de Innovación y Mejora en el Aula}

Creo que el diseño general del CIMA ha funcionado: el entusiasmo por trabajar en clase aumentó mucho durante las dos semanas, así como la participación por parte de estudiantes que en las primeras sesiones habían estado poco activos. Los dosieres de textos, junto con los videos introductorios, fueron herramientas esenciales. También lo fue la formulación de los problemas en forma de pregunta, pues parece que así su atención se centró más rápidamente en la cuestión a abordar. Las respuestas al cuestionario final estuvieron bien, no tanto por los datos cuanto por el razonamiento y reflexiones que mostraban.

Por otro lado, siendo un grupo de primer curso en el primer cuatrimestre, creo que el trabajo autónomo debería haberse combinado con pequeños momentos de explicación por mi parte. Pero planeados en forma y contenidos, y no improvisados, como ocurrió en la primera y segunda sesión. Por otro lado, para el año que viene, creo que sería conveniente adaptarlo a trabajos en grupos: como he señalado arriba, tienen una tendencia a comparar sus conclusiones que me parece que debe ser aprovechada. Para el futuro, considero que debo mejorar el diseño incluyendo momentos de explicación que no eclipsen el trabajo con fuentes, que debe ser el centro de las sesiones. No debo olvidar de que se trata de que ellos aprendan por ellos mismos, no de que yo les dé la actividad resuelta, y por tanto debo limitar mis intervenciones si quiero que este modelo funcione.

Finalmente, la principal enseñanza que extraigo de esta experiencia es la necesidad de prestar más atención a mi docencia y de tratar de modificarla progresivamente en los aspectos que no están funcionando. El diseño y aplicación del CIMA ha supuesto para mí una reflexión profunda sobre mi propia práctica, que hasta el momento solo daba resultados favorables en aquellos estudiantes que

Ciclos de Mejora en el Aula (2020). Experiencias de Innovación Docente de la US Esta obra se distribuye con la licencia Creative Commons 
mostraban una buena predisposición o unos conocimientos previos elevados. En este sentido, obligarme a reducir los contenidos a aquellos verdaderamente esenciales y tratar de reformularlos en forma de problemas vinculados a la actualidad ha sido el cambio más importante en mi docencia, y también el que ha tenido efectos más inmediatos en otras asignaturas que imparto. Igualmente, ha sido esencial para mí entender el sentido de una actividad de contraste: hasta el comienzo del CGDU, realizaba muchas actividades prácticas, pero no recapacitaba lo suficiente sobre su posible conexión con el tema a abordar. Por eso, en muchas ocasiones las prácticas que los estudiantes tenían que resolver ya estaban resueltas de antemano gracias a mi propia explicación previa. Ordenar y racionalizar este tipo de recursos y darles un sentido dentro de una secuencia más amplia es otro de los aprendizajes fundamentales de este CIMA (y del anterior) y que más han influido en mi docencia. Por último, los cuestionarios para evaluar el aprendizaje me han resultado mucho más útiles e ilustrativos de lo que esperaba. Soy consciente de que tengo que aprender a trabajar con ellos de una forma más adecuada, pero creo que en las asignaturas del segundo cuatrimestre trataré de usarlos para ajustar mejor los contenidos al nivel de los alumnos.

Palabras clave: Historia General Contemporánea, Grado en Geografía, Docencia Universitaria, Experimentación Docente Universitaria, Revoluciones Atlánticas.

Keywords: Contemporary General History, Geography Degree, University Teaching, University Teaching Experimentation, Atlantic Revolutions.

Ciclos de Mejora en el Aula (2020). Experiencias de Innovación Docente de la US Esta obra se distribuye con la licencia Creative Commons 


\section{Referencias bibliográficas}

Bain, K. (2007). Lo que hacen los mejores profesores universitarios. Valencia: Universitat de València.

De Alba, N. y Porlán, R. (2017). La metodología de enseñanza. En R. Porlán (Coord.), Enseñanza universitaria. Cómo mejorarla (pp. 38-53). Madrid: Morata.

Finkel, D. (2008). Dar clase con la boca cerrada. Valencia: Universitat de València.

García Díaz, E., Porlán, R. y Navarro, E. (2017). Los fines y los contenidos de enseñanza. En R. Porlán (Coord.), Enseñanza universitaria. Cómo mejorarla (pp. 56-72). Madrid: Morata.

Gould, E. H. y Onuf, P. S. (Coords.) (2015). Empire and Nation: The American Revolution in the Atlantic World. Baltimore: Johns Hopkins University Press.

Klooster, W. (2018). Revolutions in the Atlantic World, New Edition: A Comparative History. Nueva York: New York University Press.

Rivero, A. y Porlán, R. (2017). La evaluación de la enseñanza universitaria. En R. Porlán (Coord.), Enseñanza universitaria. Cómo mejorarla (pp. 73-118). Madrid: Morata.

Ciclos de Mejora en el Aula (2020). Experiencias de Innovación Docente de la US Esta obra se distribuye con la licencia Creative Commons 